\title{
Wireless power transfer to a micro implant device from outside of human body
}

\author{
Kazuya Yamaguchi, Kazuma Onishi, and Kenichi Iida \\ Department of Control Engineering, National Institute of Technology, Nara College
}

\section{Article Info}

\section{Article history:}

Received Jun 5, 2018

Revised Oct 11, 2018

Accepted Dec 18, 2018

\section{Keywords:}

Wireless power transfer

Biological science

State space representation

\begin{abstract}
This paper states wireless power transfer (WPT) from an AC power supply to a micro implant device in human body. At first, an equivalent circuit of WPT which contains biomedical tissue is constructed with an AC power supply, parasitic components, load resistance, and inductances. Then a state equation which stands for the behavior of circuit is found, and the expression of efficiency is derived as the ratio of the power of power supply and load. Finally an experiment is conducted based on the theoretical calculation, and the error between experimental and calculated result is computed and examined.
\end{abstract}

Copyright (c) 2019 Institute of Advanced Engineering and Science. All rights reserved.

\section{Corresponding Author:}

Kazuya Yamaguchi,

Department of Control Engineering, National Institute of Technology, Nara College,

22 Yata-cho, Yamatokoriyama, Nara, Japan.

Email: k-yamaguchi@ctrl.nara-k.ac.jp

\section{INTRODUCTION}

Wireless Power Transfer (WPT) is frequently studied and applied for various fields, for example industry, manufacture, mathematics, medical science, and information. The basic principle of transfer has been found in 19th century, although practical applied or productization is later. In 2007, a WPT system with magnetic resonance circuit whose transmitting and receiving circuits have same resonant frequency was proposed by [1]. This study accomplished highly efficient energy transfer on the situation that a power supply and load are put a few meters apart.

These days, many papers and articles have reported various WPT studies and practical products. WPT for electric vehicles is cogitated from various viewpoints, for example a design of coils [2], and incorporating solar cells [3]. Furthermore a method to drive machines is examined by actuating the rotor with piezoelectric energy via a magnetic reluctance coupling [4]. The necessity of IoT is mentioned in these days, and hence WPT adopting radio frequency is investigated to avoid replacing or recharging the batteries of wireless devices in IoT [5]. Moreover, WPT for an artificial satellite in space is tried to exchange energy wirelessly without going to space [6], and then microwave is used to send energy in greatly long distance such as this situation.

For realization to make these systems, many approaches are investigated in terms of an electric circuit, and mathematics. The resonant frequencies of all parts which compose WPT circuit are integrated, and impedance of load is matched with input impedance to maximize power of load [7]. The coils are used to transmit energy via electromagnetic field, and therefore the proper materials which are used to make high quality coils must be chosen [8]. To maximize total power of load, a mathematical model is structured based on an algorithmic study [9].

This paper focuses on the applications for medical science, especially the transmission of energy to a micro implant device from outside. The effect to human body must be considered because energy is transmitted to human body via electromagnetic field [10]. When an experiment with respect to the transmission of energy to body is conducted, an experimental animal is used, not a human body [11]. In this paper, biomedical tissue and 
a micro implant device is modeled by an electric circuit, and a mathematical equation based on modern control theory is made for finding an expression of efficiency. Moreover an experimental verification is conducted with an electric circuit whose elements have practical values, and the error between experimental and theoretical result is calculated.

\section{CALCULATION OF POWER AND EFFICIENCY}

\subsection{Design of Wireless Power Transfer Circuit Which Contains Biomedical Tissue}

In a human body, there are plasma membrane, intracellular fluid, and extracellular fluid, and they play a role different electrical characteristics each other. Current can flow in extracellular fluid, and moreover current which has high frequency can flow into intracellular fluid by going over plasma membrane. Therefore intracellular fluid and extracellular fluid have resistances $R_{\mathrm{i}}$ and $R_{\mathrm{e}}$, and plasma membrane has capacitance $C_{\mathrm{m}}$ which work as a high pass filter. In terms of this fact, these components are designed as an electric circuit as below [12].

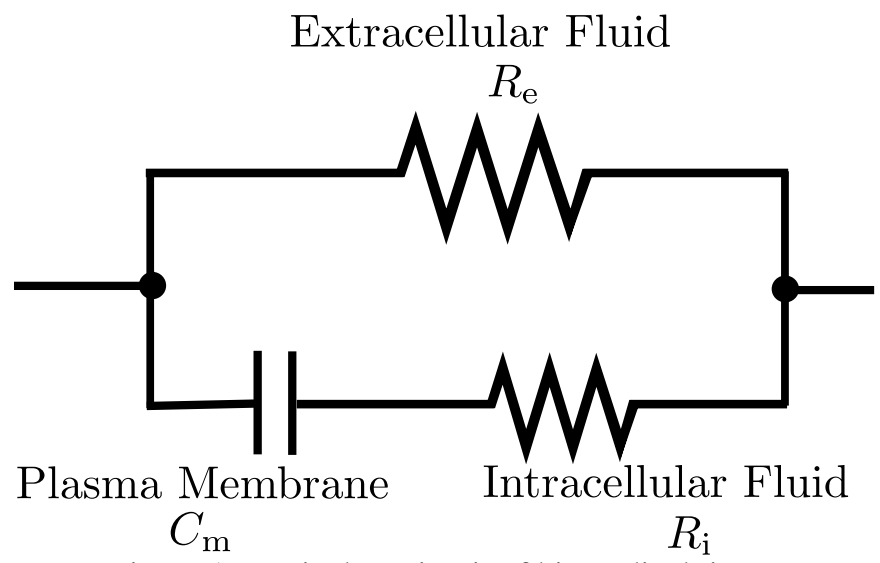

Figure 1. Equivalent circuit of biomedical tissue

With Figure 1, a wireless power transfer circuit for charging a micro implant device is designed as follows.

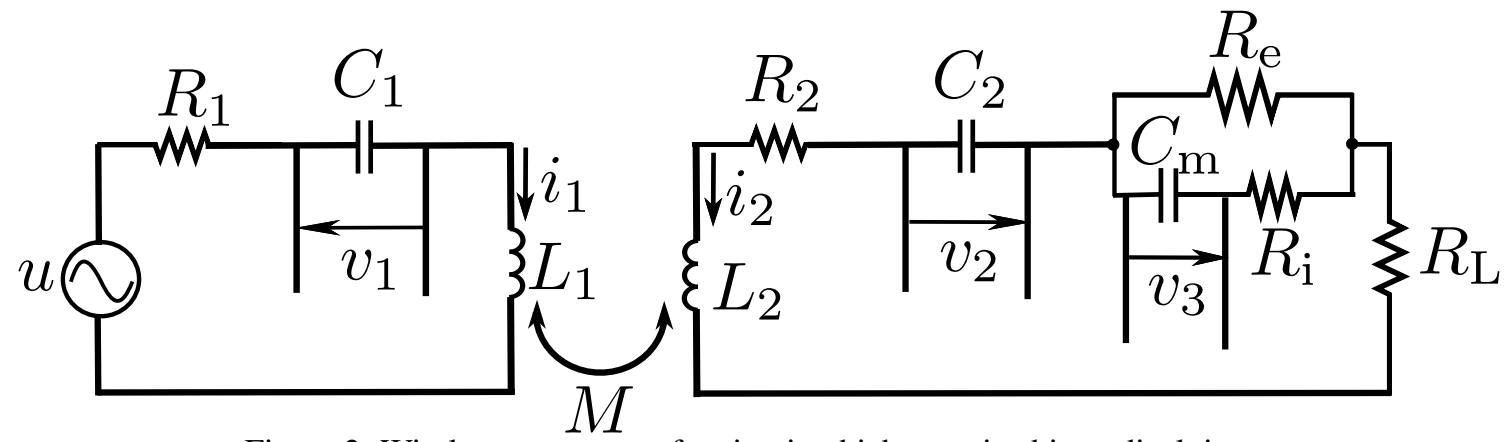

Figure 2. Wireless power transfer circuit which contains biomedical tissue

In Figure 2, the left side is a transmitting circuit, and the right side is equivalent circuit which contains biomedical tissue and the micro implant device. $u$ is the voltage of power supply, $R_{1}, R_{2}, C_{1}, C_{2}$ are parasitic components, $L_{1}, L_{2}$ are the antennas, $M$ is mutual inductance, and $R_{\mathrm{L}}$ is the micro implant device. 
2.2. Modeling of Wireless Power Transfer Circuit by Using a State Equation which has State Variables Voltage and Current

From Figure 2, a state equation which has state variables voltage and current is obtained as below [13].

$$
\begin{aligned}
\dot{x} & =A x+B u, x=\left[\begin{array}{lllll}
v_{1} & v_{2} & v_{3} & i_{1} & i_{2}
\end{array}\right]^{\mathrm{T}} \\
A & =\frac{1}{\Delta}\left[\begin{array}{ccccc}
0 & 0 & 0 & \frac{\Delta}{C_{1}} & 0 \\
0 & 0 & 0 & 0 & \frac{\Delta}{C_{2}} \\
0 & 0 & -\frac{\Delta}{\left(R_{\mathrm{i}}+R_{\mathrm{e}}\right) C_{\mathrm{m}}} & 0 & \frac{R_{\mathrm{e}} \Delta}{\left(R_{\mathrm{i}}+R_{\mathrm{e}}\right) C_{\mathrm{m}}} \\
-L_{2} & M & \frac{R_{\mathrm{e}} M}{R_{\mathrm{i}}+R_{\mathrm{e}}} & -R_{1} L_{2} & \frac{\left[R_{3}\left(R_{\mathrm{i}}+R_{\mathrm{e}}\right)+R_{\mathrm{i}} R_{\mathrm{e}}\right] M}{R_{\mathrm{i}}+R_{\mathrm{e}}} \\
M & -L_{1} & -\frac{R_{\mathrm{e}} L_{1}}{R_{\mathrm{i}}+R_{\mathrm{e}}} & R_{1} M & -\frac{\left[R_{3}\left(R_{\mathrm{i}}+R_{\mathrm{e}}\right)+R_{\mathrm{i}} R_{\mathrm{e}}\right] L_{1}}{R_{\mathrm{i}}+R_{\mathrm{e}}}
\end{array}\right], B=\frac{1}{\Delta}\left[\begin{array}{c}
0 \\
0 \\
0 \\
L_{2} \\
-M
\end{array}\right] \\
\Delta & =L_{1} L_{2}-M^{2}, R_{3}=R_{2}+R_{\mathrm{L}} .
\end{aligned}
$$

\subsection{Definition and calculation of power and efficiency}

The vector of stationary solution $x_{\mathrm{ss}}$ which is composed of voltage $v_{1}, v_{2}, v_{3}$ and current $i_{1}, i_{2}$ is found by solving the state equation (1).

$$
x_{\mathrm{sS}}(t)=\alpha \cos \omega t+\beta \sin \omega t
$$

where $\alpha=-\omega\left(\omega^{2} I+A^{2}\right)^{-1} B, \beta=-A\left(\omega^{2} I+A^{2}\right)^{-1} B$, and $\omega$ is angular frequency of $u$. Moreover column vectors $\alpha$ and $\beta$ are expressed in the following.

$$
\alpha=\left[\begin{array}{lllll}
\alpha_{1} & \alpha_{2} & \alpha_{3} & \alpha_{4} & \alpha_{5}
\end{array}\right]^{\mathrm{T}}, \beta=\left[\begin{array}{lllll}
\beta_{1} & \beta_{2} & \beta_{3} & \beta_{4} & \beta_{5}
\end{array}\right]^{\mathrm{T}}
$$

Power transmission efficiency $\eta_{\text {load }}$ and energy loss efficiency $\eta_{\text {loss }}$ are expressed as follows.

$$
\begin{aligned}
\eta_{\text {load }} & =\frac{P_{\text {load }}}{P_{\text {in }}}=\frac{R_{\mathrm{L}}\left(\alpha_{5}^{2}+\beta_{5}^{2}\right)}{\beta_{4}} \\
\eta_{\text {loss }} & =\frac{P_{\text {loss }}}{P_{\text {in }}}=\frac{\omega^{2}\left(R_{\mathrm{i}}-R_{\mathrm{e}}\right)\left(\alpha_{3}^{2}+\beta_{3}^{2}\right) C_{\mathrm{m}}^{2}-2 \omega R_{\mathrm{e}} C_{\mathrm{m}}\left(\alpha_{3} \beta_{5}-\alpha_{5} \beta_{3}\right)-R_{\mathrm{e}}\left(\alpha_{5}^{2}+\beta_{5}^{2}\right)}{\beta_{4}}
\end{aligned}
$$

where $P_{\text {in }}$ is power of $u, P_{\text {load }}$ is power of $R_{\mathrm{L}}$, and $P_{\text {loss }}$ is power of $R_{\mathrm{i}}$ and $R_{\mathrm{e}}$.

\section{EXPERIMENTAL VERIFICATION}

3.1. Condition of experiment for wireless power transfer by using the equivalent circuit to suppose power transmission for a micro implant device in human body from outside

The experimental circuit is shown in Figure 3.

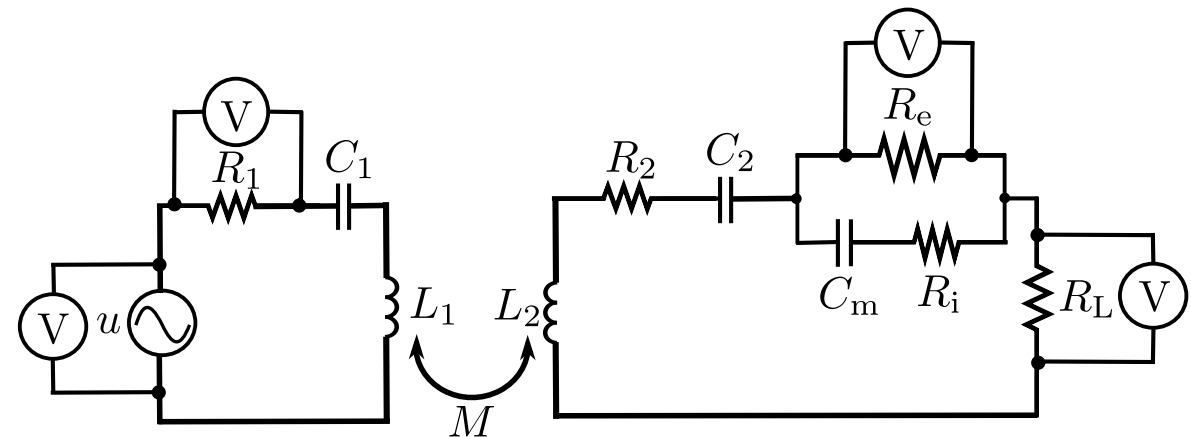

Figure 3. Experimental circuit 
The values of elements are shown in Table 1.

Table 1. Values of Elements

\begin{tabular}{cccccc}
\hline \multicolumn{5}{c}{ Values of Elements } \\
\hline$R_{1}$ & $465 \Omega$ & $R_{2}$ & $10.0 \Omega$ & $R_{3}$ & $97.4 \Omega$ \\
$R_{\mathrm{i}}$ & $684 \Omega$ & $R_{\mathrm{e}}$ & $5.37 \mathrm{k} \Omega$ & & \\
$L_{1}$ & $66.0 \mu \mathrm{H}$ & $L_{2}$ & $66.4 \mu \mathrm{H}$ & $M$ & $9.27 \mu \mathrm{H}$ \\
$C_{1}$ & $84.0 \mathrm{pF}$ & $C_{2}$ & $85.5 \mathrm{pF}$ & $C_{\mathrm{m}}$ & $11.7 \mathrm{nF}$ \\
\hline
\end{tabular}

\subsection{Variation of efficiency versus frequency of a power supply}

The variation of efficiency is investigated by changing frequency of the power supply $u$. The calculated and experimental results are shown in Figure 4.

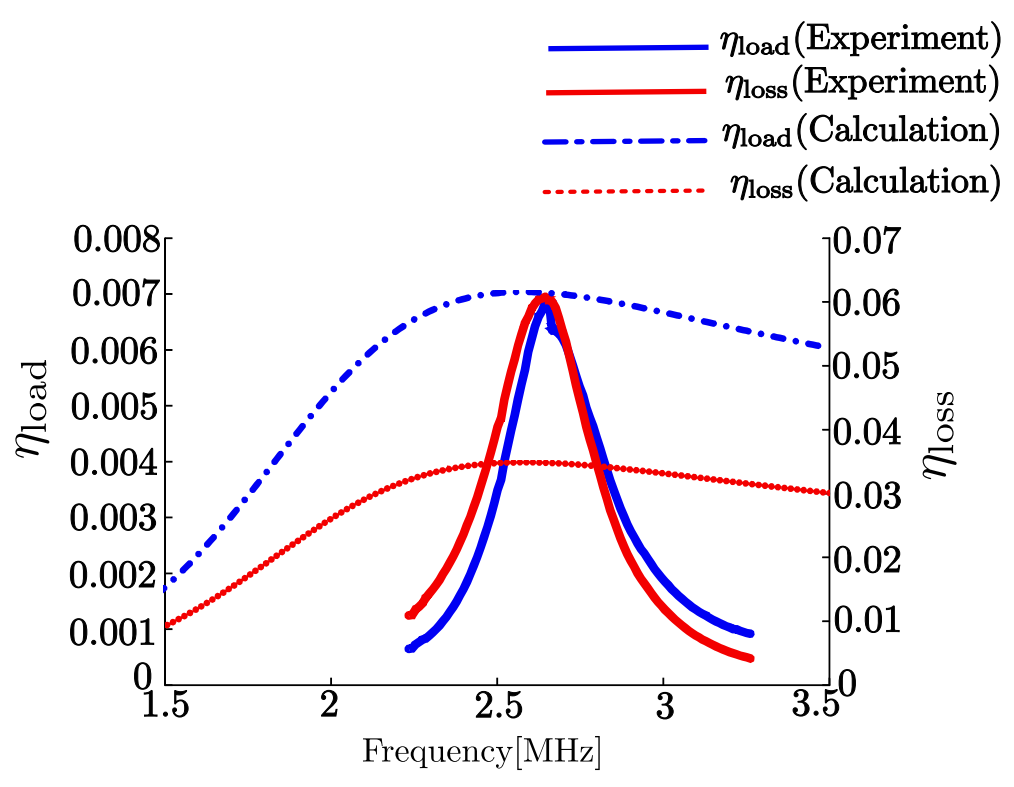

Figure 4. Variation of efficiency versus frequency of a power supply

\subsection{Discussion}

Figure 4 shows that the optimal frequency which maximizes efficiency is $f_{\text {opt }}=2.65[\mathrm{MHz}]$, and the error between calculation and experiment is shown on Table 2.

Table 2. Efficiency and Error at the Optimal Frequency $f_{\mathrm{opt}}=2.65[\mathrm{MHz}]$

\begin{tabular}{ccc}
\hline & $\eta_{\text {load }}$ & $\eta_{\text {loss }}$ \\
\hline Experiment & $6.85 \times 10^{-3}$ & $6.05 \times 10^{-2}$ \\
Calculation & $7.08 \times 10^{-3}$ & $3.41 \times 10^{-2}$ \\
Error[\%] & -3.19 & +77.6 \\
\hline
\end{tabular}

With respect to $\eta_{\text {load }}$, the experimental maximum value is almost same as theoretical value which is found by equation (3). On the other hand, the experimental maximum $\eta_{\text {loss }}$ is greatly different for calculation. This error is caused by the approximation which depends on frequency and impedance. 


\section{CONCLUSION}

This study tried to inspect transmission of energy to a micro implant device wirelessly. An equivalent circuit which supposes the micro implant device and biomedical tissue was designed, and an equation was found for calculating power and efficiency. Moreover an experiment was performed to prove the appropriateness of calculation and detect an error and cause of it.

As an approach to consider effective situation furthermore, a meat of animals should be used as a load resistance. The influence of electromagnetic field must be considered for making some productions which charge a micro implant device through human body.

\section{REFERENCES}

[1] A. Kurs, A. Karalis, R. Moffatt, J. D. Joannopoulos, P. Fisher, and M. Soljačić, "Wireless Power Transfer via Strongly Coupled Magnetic Resonances”, Science, vol. 317, pp. 83-86, 2007.

[2] S. Chatterjee, A. Iyer, C. Bharatiraja, I. Vaghasia, and V. Rajesh, "Design Optimisation for an Efficient Wireless Power Transfer System for Electric Vehicles”, Energy Procedia, vol. 117, pp. 1015-1023, 2017.

[3] H. Pan, L. Qi, X. Zhang, Z. Zhang, W. Salman, Y. Yuan, and C. Wang, "A portable renewable solar energy-powered cooling system based on wireless power transfer for a vehicle cabin", Applied Energy, vol. 195, pp. 334-343, 2017.

[4] P. Pillatsch, E. M. Yeatman, A. S. Holmes, and P. K. Wright, "Wireless power transfer system for a human motion energy harvester", Sensors and Actuators A: Physical, vol. 244, pp. 77-85, 2016.

[5] L. Han, and L. Li, "Integrated wireless communications and wireless power transfer: An overview", Physical Communication, vol. 25, part 2, pp. 555-563, 2017.

[6] M- L. Zhong, Y- Z. Li, Y- F. Mao, Y- H. Liang, and J. Liu, "Coupled optic-thermodynamic analysis of a novel wireless power transfer system using concentrated sunlight for space applications", Applied Thermal Engineering, vol. 115, pp. 1079-1088, 2017.

[7] M. Rentschler, and I. Bhattacharya, "Decoupled control of wireless power transfer: Eliminating the interdependence of load resistance and coupling to achieve a simple control framework with fast response times", International Journal of Electrical Power \& Energy Systems, vol. 99, pp. 156-163, 2018.

[8] X. Wang, X. Nie, Y. Liang, F. Lu, Z. Yan, and Y. Wang, "Analysis and experimental study of wireless power transfer with HTS coil and copper coil as the intermediate resonators system", Physica C: Superconductivity and its Applications, vol. 532, pp. 6-12, 2017.

[9] I. Katsidimas, S. Nikoletseas, T. P. Raptis, and C. Raptopoulos, "An algorithmic study in the vector model for Wireless Power Transfer maximization", Pervasive and Mobile Computing, vol. 42, pp. 108-123, 2017.

[10] J. Y. Mun, M. G. Seo, W. G. Kang, H. Y. Jun, Y. H. Park, and J. K. Pack, "Study on the Human Effect of a Wireless Power Transfer Device at Low Frequency”, PIERS Proceedings, pp. 322-324, 2012.

[11] C- W. Chang, K- C. Hou, and L- J. Shieh, "Wireless powering electronics and spiral coils for implant microsystem toward nanomedicine diagnosis and therapy in free-behavior animal", Solid- State Electronics, pp. 93-100, 2012.

[12] T. Kinoshita, "Measurement of Body Impedance - Waveform Measurement using a Visual Programming Language -".

[13] K. Yamaguchi, Y. Yamamoto, T. Hirata, E. Setiawan, and I. Hodaka, "Mathematical Expression of Optimal Frequencies for Wireless Power Transfer", Proceedings of The 3rd International Conference on Computer Engineering \& Mathematical Sciences, pp. 826-827, 2014. 